\title{
Dissolution DNP for in vivo preclinical studies
}

\author{
Arnaud Comment ${ }^{1,2}$ \\ ${ }^{1}$ Institute of Physics of Biological Systems, Ecole Polytechnique Fédérale de \\ Lausanne, CH-1015 Lausanne, Switzerland \\ ${ }^{2}$ General Electric Healthcare, Pollards Wood, Nightingales Lane, Chalfont St Giles, \\ Buckinghamshire, HP8 4SP, United Kingdom
}

arnaud.comment@epfl.ch; arnaud.comment@ge.com

Keywords: Hyperpolarization; Dynamic Nuclear Polarization; Magnetic Resonance;

MRI; Molecular Imaging. 


\begin{abstract}
The tremendous polarization enhancement afforded by dissolution dynamic nuclear polarization (DNP) can be taken advantage of to perform preclinical in vivo molecular and metabolic imaging. Following the injection of molecules that are hyperpolarized via dissolution DNP, real-time measurements of their biodistribution and metabolic conversion can be recorded. This technology therefore provides a unique and invaluable tool for probing cellular metabolism in vivo in animal models in a noninvasive manner. It gives the opportunity to follow and evaluate disease progression and treatment response without requiring ex vivo destructive tissue assays. Although its considerable potential has now been widely recognized, hyperpolarized magnetic resonance by dissolution DNP remains a challenging method to implement for routine in vivo preclinical measurements. The aim of this article is to provide an overview of the current state-of-the-art technology for preclinical applications and the challenges that need to be addressed to promote it and allow its wider dissemination in the near future.
\end{abstract}




\section{Introduction}

New technologies often create two clashing waves of over-optimism and skepticism. Hyperpolarized magnetic resonance (MR) was clearly no exception to this rule. Many among the early pessimists have now changed their mind and some of the overenthusiastic medical and biomedical researchers were somewhat disappointed by the unanticipated difficulties. However, the preclinical studies that are ongoing at the time I am writing this manuscript are expected to further demonstrate that it is possible to noninvasively detect biological processes in vivo in a way that only true believers had anticipated.

Today, it therefore seems quite straightforward to answer the question raised at the 2008 annual congress of the European Society for Magnetic Resonance in Medicine and Biology (ESMRMB, Valencia, Spain) during the roundtable discussion, namely "Hyperpolarization or hype and polarization?". Hopefully, this perspective article will convince the outstanding pessimists that they should consider investing in this technology, in particular in dissolution dynamic nuclear polarization (DNP) for preclinical applications.

\section{Hyperpolarization by dissolution DNP in a nutshell}

Although this is most likely the section you may want to skip if you are already familiar with this technology, I will attempt to describe what hyperpolarization is as briefly as possible and review part of the jargon that has been established in the field.

The term hyperpolarization was originally introduced in the MR community by researchers working on noble gas MRI [1] , notably ${ }^{3} \mathrm{He}$ and ${ }^{129} \mathrm{Xe}$. When it relates to liquid-state NMR, hyperpolarized refers to a nuclear spin state that is obtained after 
either (1) a rapid and large temperature jump through dissolution DNP [2] or the brute force method, which has also been proposed for hyperpolarizing noble gases [3], or (2) spin order transfer from parahydrogen through a method called parahydrogeninduced nuclear polarization (PHIP) [4-6]. While the brute force method only relies on the Boltzmann polarization that is obtained after reaching thermal equilibrium at low temperature and high magnetic field, dissolution DNP makes use of electron spins to dynamically boost the nuclear polarization up to a very high level without having to reach temperatures below about $1 \mathrm{~K}$. The PHIP method also relies on low temperature to favor a specific spin state of dihydrogen, namely the parahydrogen singlet state, but the target molecule that needs to be polarized for the MR experiments does not experience any temperature jump. Instead, a room-temperature chemical reaction transfers spin order from parahydrogen to the molecule of interest and the insensitive nuclei (typically ${ }^{13} \mathrm{C}$ ) located in the molecule are subsequently polarized via a low-field polarization transfer [7].

The highest liquid-state polarizations that have been reported so far were obtained using dissolution DNP $[8,9]$. The principle of DNP is to make use of the much larger gyromagnetic ratio of electron spins to enhance the nuclear spin polarization through cross-polarization driven by a slightly off-resonance irradiation of the ESR line. Two fundamental physical parameters make it possible to obtain nuclear polarizations close to unity in a field of $3 \mathrm{~T}$ or above and at a temperature of about $1 \mathrm{~K}$ : (a) the electron spin polarization is essentially 1 (Fig.1), and (b) the electron spin relaxation is long enough to allow the saturation of the ESR and hence promote crosspolarization with adjacent nuclear spins (Fig.2). The unpaired electron spins, which are also referred to as polarizing agents in the context of dissolution DNP, are usually introduced in the form of persistent radicals into the sample prior to cooling and 
solidifying the sample in a glassy form. To date, the most efficient polarizing agents are trityl or 1,3-bisdiphenylene-2-phenylallyl (BDPA) radicals [2, 10], although nitroxyl radicals have also been used for in vivo preclinical work [11-13], and a new type of non-persistent photo-induced radicals show promising features [14, 15].

The original hyperpolarizer was operating at $3.35 \mathrm{~T}$ [2], but it was later shown that larger polarizations can be obtained in a field of $5 \mathrm{~T}[16,17]$ or $7 \mathrm{~T}[18]$. As a rule of thumb, at least for samples containing wide-ESR-line-width polarizing agents such as nitroxyl radicals, the maximum polarization that can be achieved at a working temperature of about $1 \mathrm{~K}$ is inversely proportional to the temperature [19]. Therefore, within the parameter space explored so far by the researchers working on dissolution DNP, increasing the field and lowering the temperature will lead to larger polarizations. Note that the operating field and temperature also strongly affect the dynamics of DNP and the polarization build-up times will also increase with increasing field and decreasing temperature.

\section{What unique information can be gathered using in vivo hyperpolarized MR}

Before going further into some of the technical aspects of the dissolution DNP method, I would like to give a succinct overview of the assets of hyperpolarized MR in the context of in vivo preclinical applications.

Hyperpolarized MR provides a unique way to measure metabolism in real time. No other technique allows probing metabolic transformations in vivo with the time resolution of one second. The essential point is that the largely enhanced nuclear polarization affords sufficient sensitivity to acquire, in addition to the spectral information that allows distinguishing the various metabolites, high-spatial and high- 
temporal resolution data. Therefore, metabolic pools of relatively small size can be detected in specific areas of the body under investigation with high temporal resolution. It must be underlined that the spatial and temporal resolutions are nevertheless both restricted by the available signal-to-noise ratio (SNR): the higher the spatial resolution, the lower the temporal resolution, and vice versa.

Several recent reviews have covered the various preclinical applications performed to date with the most prominent metabolic precursors $[20,21]$, in particular in the context of cancer research $[22,23]$, and I refer the readers to these articles if they wish to have a complete view of the current state-of-the-art biomedical applications. In the present perspective article, I will focus on one of the most interesting yet challenging substrate, namely ${ }^{13} \mathrm{C}$-glucose. It will be taken as case study to highlight the shortcomings of dissolution DNP and discuss how to address them.

Only one in vivo metabolic study with hyperpolarized ${ }^{13} \mathrm{C}$-glucose has been so far published [24], but it is certainly not because of a lack of interest or relevance. Glucose is a substrate of major interest for at least two main reasons: first, because it is an essential fuel for mammals, in particular for their brain; second, because upregulated glycolysis is a hallmark of most cancer cells. In fact, the latter partially explains why the first in vivo results were obtained in a cancer model [24]. The first in vitro experiments have already been performed several years ago in E. coli and yeast $[25,26]$, and more recently in cancer cells $[27,28]$. However, in order to achieve the necessary sensitivity to generally observe glucose metabolites in vivo in rodents, several challenges must be overcome.

Positron emission tomography (PET) following the administration of the ${ }^{18} \mathrm{~F}$ fluorodeoxyglucose glucose analog (FDG) is currently considered as one of the most 
established modalities to detect tumors and to evaluate their progression and the impact of treatments. FDG-PET provides information on glucose uptake, but it does not allow measuring the fate of glucose once it has entered the cells and undergone the phosphorylation process catalyzed by hexokinase. Hyperpolarized MR therefore brings a unique way of performing true metabolic imaging since it will report on glycolysis well beyond cellular uptake and yield spatiotemporal information on the metabolites deriving from the precursor. An important downstream metabolite of glucose for the detection of disease, notably cancer, is lactate. Higher lactate is indeed a consequence of the so-called Warburg effect [29-31]. Hyperpolarized [1${ }^{13} \mathrm{C}$ ]pyruvate has been used as a surrogate for glucose to detect this effect. The recent studies combining FDG-PET and hyperpolarized ${ }^{13} \mathrm{C}$-pyruvate MR experiments in the same subjects demonstrated the added value of hyperpolarized MR for assessing glycolytic metabolism [32-34].

Pyruvic acid not only plays a crucial role in mammalian metabolism, but it can also be qualified as the wonder molecule for hyperpolarized MR by dissolution DNP. It is a highly-concentrated self-glassy compound with a long ${ }^{13} \mathrm{C} \mathrm{T}_{1}$ (on two out of its three carbon sites) that is only slightly field-dependent, and it was even recently shown to be photo-excitable to generate in situ radicals that can be used for DNP [15]. Glucose does not exhibit any of those key features.

Although lactate has proven to be a valuable metabolite for evaluating glycolytic metabolism in tumors, other metabolites have been observed using hyperpolarized [1${ }^{13}$ ]glucose, notably $6 \mathrm{PG}$, an intermediate of the pentose phosphate pathway (see Fig.3). The detection of such a metabolic pathway in vivo has never been done and is perhaps one of the key for probing mechanisms responsible for tumor progression, 
e.g. NADPH production [35]. This might be one of the most insightful probe for evaluating the activity of cancer cells.

Another promising substrate that has been recently proposed to probe glycolysis intermediates is ${ }^{13} \mathrm{C}$-dihydroxyacetone which, after being phosphorylated, exchange with glyceraldehyde 3-phosphate [36]. In addition to pyruvate, lactate, and alanine, Moreno et al. observed the real-time formation of 3-phosphoglycerate (3-PG) and phosphoenolpyruvate (PEP) in perfused mouse liver (see Fig.4). The ${ }^{13} \mathrm{C} \mathrm{T}_{1}$ relaxation time of $\left[2-{ }^{13} \mathrm{C}\right] \mathrm{DHA}$ at $9.4 \mathrm{~T}$ was reported to be $32 \mathrm{~s}$, which is a big asset for this substrate. Indeed, even when deuterated, the main issue with hyperpolarized substrates such as glucose is that their longitudinal relaxation time is quite short (about $10 \mathrm{~s}$ in vitro at $14.1 \mathrm{~T}$ [26], and $9 \mathrm{~s}$ in vivo at $7 \mathrm{~T}$ [24]), especially at low magnetic field.

Molecular imaging based on hyperpolarized MR with inert and non-metabolizable substances such as ${ }^{6}$ Li-lithium salt [37], $\left[{ }^{13} \mathrm{C}\right]$ urea $[38], \quad\left[{ }^{13} \mathrm{C}\right]$ hydroxymethyl cyclopropane [39], or $\left[{ }^{13} \mathrm{C}\right]$ tert-butanol [40], has also been proposed as a modality for in vivo preclinical applications. It was for instance shown by Reed et al. [41] that the large SNR can lead to spectacular high-resolution ${ }^{13} \mathrm{C}$ MR angiography (see Fig.5). Note that it has been shown that DNP can also be used to hyperpolarize ${ }^{129} \mathrm{Xe}[42]$, a gas that has been already used for perfusion imaging in vivo [43].

\section{The nuts and bolts of dissolution DNP}

The overall challenge associated with in vivo hyperpolarized MR can be summarized in a single question: how to obtain the highest in vivo SNR from the time the hyperpolarized molecules have reached the targeted organ or cells? 
Any preclinical hyperpolarized MR experiment can be broken down in three key steps: (a) dissolution DNP, (b) transfer and injection of hyperpolarized molecules, and (c) in vivo MR acquisition. I will now discuss in some details the challenges associated with each one of these three steps.

\section{a. Dissolution DNP}

The aim of the DNP part of this key step is essentially identical to the one originally set by the particle physicists working on solid polarized targets: to reach the highest solid-state nuclear polarization. One strategy to obtain the highest polarization was to cool the sample to sub-Kelvin temperatures. Two main particularities defined the new frontier set by dissolution DNP and imposed new challenges that had not been tackled by the polarized target community: (1) the nuclear spins that need to be polarized are neither protons nor deuterons, as was usually the case for polarized targets, but rather larger atoms such as ${ }^{13} \mathrm{C}$ or ${ }^{15} \mathrm{~N}$, and (2) once polarized, the sample has to be extracted from the cryogenic environment, which essentially precludes the introduction of the target inside the closed-cycle cryogenic system of complex apparatuses such as ${ }^{3} \mathrm{He}-$ ${ }^{4} \mathrm{He}$ dilution refrigerators. Hence, if we also take into account the global scarcity of ${ }^{3} \mathrm{He}$, a DNP system compatible with dissolution should operate with ${ }^{4} \mathrm{He}$ only. Thanks to the development of narrow-ESR-line radicals $[10,44]$, it is possible to polarize low-gamma nuclei such as ${ }^{13} \mathrm{C}$ and ${ }^{15} \mathrm{~N}$ without polarizing ${ }^{1} \mathrm{H}$, which greatly enhances the efficiency of the process and allows reaching ${ }^{13} \mathrm{C}$ polarization up to $60-70 \%$ even at temperatures as "high" as $1 \mathrm{~K}[9,17]$. Another practical constraint imposed by dissolution DNP that is less relevant for the polarized target community, is the need to limit the polarization time in order to reach a high throughput. A delay on the order of an hour between each experiment would be highly desirable. Technical adaptations to polarize multiple samples were proposed and allowed the production of up to 4 
hyperpolarized samples within an hour with a minimum delay of 5-15 min between each dissolution [8, 45], which should be suitable for most applications. Another possible route for accelerating the polarization process is to make use of ${ }^{1} \mathrm{H}^{-13} \mathrm{C}$ or ${ }^{1} \mathrm{H}$ -

${ }^{15} \mathrm{~N}$ cross-polarization $(\mathrm{CP})$ since it was shown that ${ }^{1} \mathrm{H}$ polarize faster than ${ }^{13} \mathrm{C}$ or ${ }^{15} \mathrm{~N}$ with wide-ESR-line radicals [46]. A low-temperature CP scheme compatible with dissolution has recently been implemented and it was shown that up to $25 \%{ }^{13} \mathrm{C}$ liquid-state polarization could be obtained within 20 min [47].

All the critical features required for high throughput of highly polarized samples have been implemented in the state-of-the-art polarizer designed by Ardenkjaer-Larsen et al. for clinical intent (see Fig.6) [48], and this instrument has led to the development of the SpinLab ${ }^{\mathrm{TM}}$, a product commercialized by GE Healthcare. To date, no commercial hyperpolarizer dedicated and optimized for preclinical studies has ever been implemented, and it is not always straightforward to compare the performances of the various systems that have been used for this purpose. While many research groups are still working on finding ways to improve the solid-state polarization, it is the author's opinion that the crucial point for preclinical applications is not so much to increase the maximum solid-state polarization, the established methods having already been shown to lead to remarkably high levels, but rather to reach the highest liquid-state polarization at the time of injection.

\section{b. Transfer and injection}

Dissolution DNP being an ex situ polarization technique, i.e. the polarization process does not take place in the environment where the MR acquisition is performed, the hyperpolarized molecules have to be transferred from inside the polarizer to the bore of the MR instrument. Because the hyperpolarized state of the spins under 
investigation is a highly out-of-equilibrium state, the challenge is to maintain the high polarization created through dissolution DNP until the molecules reach their target. The low magnetic field experienced by the molecules during the transfer usually leads to increased relaxation rates. The goal of this key step is to implement a nearly lossless sample transfer and an injection apparatus that can maintain the hyperpolarized molecules in optimal conditions to minimize relaxation prior and during the injection. However, because relaxation is unavoidable, the crucial parameter that first needs to be minimized is the transfer time. Cheng et al. have implemented a method to set a delay as short as $3 \mathrm{~s}$ between dissolution and injection [49]. It is based on a dedicated injection pump specifically designed to decouple the high-speed transfer process from the physiologically-compatible injection rate (see Fig. 7) [19]. This instrument has proven to be of high value for several preclinical studies because it allows working with short- $\mathrm{T}_{1}$ compounds, such as the mediumchain fatty acid ${ }^{13}$ C-octanoate [50]. Mishkovsky et al. reported a liquid-state polarization larger than $20 \%$ in $\left[\mathrm{U}_{-}{ }^{2} \mathrm{H}, \mathrm{U}_{-}{ }^{13} \mathrm{C}\right]$ glucose at the time of injection using this method [51]. Note that this injection pump not only allows reducing the transfer time and maintaining the sample in a high-field environment, which in most cases will minimize the losses due to relaxation, it also allows optimizing the temperature of the sample during the injection [49].

Another way to reduce the polarization losses during the transfer is to eliminate the presence of paramagnetic impurities used for the DNP process, namely radicals and/or Gd complexes. If DNP is performed using nitroxyl radicals, they can be scavenged using ascorbic acid to minimize the losses [49, 52]. They can also be trapped in a hydrogel or a solid-matrix $[53,54]$. Perhaps the cleanest way to avoid any kind of contamination and completely eliminate the need for a filtering procedure is the use of 
photo-induced radicals that recombine upon dissolution [15]. They have also been shown to lead to reduced polarization losses when the hyperpolarized solution crosses complex magnetic field paths [14].

Due to the limited lifetime of the hyperpolarized state, a fast injection is desirable, but the injection rate is obviously limited by physiological considerations. The volume of the injected solution is also limited and depends on the blood volume of the animal under investigation. In some cases, e.g. pyruvic acid, the polarized sample is highly concentrated and it is necessary to highly dilute it prior to injection (the concentration of neat pyruvic acid is $14.4 \mathrm{M}$, while the typical pyruvate concentration range used for in vivo application is $100-250 \mathrm{mM}$, for doses ranging from 0.1 to $0.5 \mathrm{mmol} / \mathrm{kg}$ ). In other cases, the concentration of the sample after dissolution is lower than it could be in terms of physiology. This is the case for glucose solutions (typically not above 200 $\mathrm{mM}$ ), and a higher concentration would be desirable because the bolus volume is limited.

\section{c. In vivo MR acquisition}

Once the intravenous injection has been executed, little or nothing can be done to restrict the longitudinal relaxation of the hyperpolarized molecules circulating in blood and tissue. In addition to the inherent longitudinal relaxation, the application of radiofrequency (rf) pulses required for MR acquisition will also participate in the decay of the enhanced nuclear polarization. One critical element in this key step is therefore to use an adapted MR acquisition scheme for optimizing the SNR while gathering the data of interest. Before introducing the different imaging schemes that have been developed for hyperpolarized MR, I would like to briefly discuss, in the 
context of a simple acquisition of a temporal series of unlocalized spectra, the decisive parameters that should be accounted for.

In the framework of in vivo hyperpolarized MR studies with $\left[1-{ }^{13} \mathrm{C}\right]$ pyruvate or $[1-$ ${ }^{13} \mathrm{C}$ ]acetate, it was demonstrated that an analysis based on the relative ratio between substrate and metabolites can provide the same kinetic information as an analysis based on the evolution of the substrate and metabolite signals [55, 56]. Although this will not always hold true, it shows that in some cases a scheme based on the maximization of the SNR of the summed spectra can be advantageous. To illustrate how this can be optimized, a simulation of the total SNR as a function of the various acquisition parameters is presented in Fig. 8. It is assumed that the $T_{1}$ is $9 \mathrm{~s}$, which corresponds the in vivo ${ }^{13} \mathrm{C}_{1}$ of $\left[\mathrm{U}_{-}{ }^{2} \mathrm{H}\right.$, U- $\left.{ }^{13} \mathrm{C}\right]$ glucose determined by Rodrigues et al. [24]. It can be seen that, for such a short $\mathrm{T}_{1}$, it can be highly beneficial to increase the flip angle and reduce the delay between each acquisition. Another scheme consisting in increasing the flip angle of the excitation pulse over the acquisition period has been proposed to improve the SNR of hyperpolarized MR [57]. It must also be noted that the high SNR together with the inherent sparsity of hyperpolarized ${ }^{13} \mathrm{C}$ MR spectra, which typically contain a limited number of peaks, are suitable for applying compressed sensing method to accelerate in vivo data acquisition [58].

Many in vivo studies have been performed using either single-voxel spectroscopy [5961], or have relied on the restricted field of view (FOV) of a surface coil to localize the signal $[55,62,63]$. For imaging, two main schemes have been proposed to individually follow the evolution of the metabolites within a slice of interest: either apply a broadband excitation and record, at a series of given time points, the entire NMR spectrum in each voxel using fast acquisition schemes [64], or apply selective excitation at each frequency corresponding to a set of given metabolites prior to consecutively record images in the spatial dimensions only [15, 65-67]. The second approach has the advantage of an increased spatial resolution and would therefore be 
preferable if the discrete number of metabolites of interest is limited and their resonances are spectrally well separated to allow sufficient selectivity in the excitation. Note that an elegant variant of the first scheme, based on the use of spatiotemporal encoding (SPEN), has also been used for in vivo metabolic imaging [68].

Because of the transient nature of the hyperpolarized signals and because many measurements are based on single-shot acquisitions, it is also crucial to perform hyperpolarized MR experiments with highly sensitive rf probes. Recent advances in coil design have allowed improving the SNR of ${ }^{13} \mathrm{C}$ MR experiments $[69,70]$, and preliminary data obtained at high field with cryogenic probes shows interesting promise [71]. Surface coils provide the highest sensitivity thanks to their proximity to the tissue under investigation, but it must be noted that for acquiring artifact-free images using complex excitation schemes, it might be preferable to use a volume coil for excitation. Optimization of the MR parameters has for instance led to the acquisition of state-of-the-art ${ }^{13} \mathrm{C}$ metabolic images allowing the monitoring of mutant IDH1 activity in glioma in vivo (see Fig. 9) [72].

Since the nuclear polarization is determined by the dissolution DNP process rather than by the measuring field, it may be tempting to deduce that there is no advantage in working at high field and that low-field bench top MR scanners might provide the ideal solution for hyperpolarized MRI. However, another crucial aspect that is inherent to all measurements based on spectroscopy is the necessity to acquire sharp and well separated resonances, which reveals the benefit of both high field and highorder shimming. Not only will broader lines lead to poorer SNR, but they may also overlap with neighboring resonances and preclude the detection of important 
metabolites. The addition of a proton decoupling scheme will also improve the line width of the resonance. Hyperpolarized ${ }^{13} \mathrm{C}$ line widths as narrow as $6 \mathrm{~Hz}$ have been measured in in vivo experiments performed at $9.4 \mathrm{~T}$ [61]. An example of complex spectrum requiring high field, high-order shimming, and proton decoupling to resolve the ${ }^{13} \mathrm{C}$ resonances of a set metabolites measured in vivo in the rat heart following the co-injection of two substrates, namely $\left[1-{ }^{13} \mathrm{C}\right]$ pyruvate and $\left[1-{ }^{13} \mathrm{C}\right]$ butyrate, is shown in Fig. 10.

\section{Limitations and future directions}

Several important points should be highlighted to understand the limitations of the technique and how it can be improved in the future. Some are linked to physiological constraints that cannot be alleviated. The central question is whether or not the recorded data is impaired by the measurements, in particular because in most cases supraphysiological doses will be injected. PET agents are called "tracers" because they are literally injected in traces amount. This is not the case with hyperpolarized agents since the typical blood concentration after bolus injection in a rodent will be in the $\mathrm{mM}$ range. It is therefore fair to raise the question of the relevancy of the measurements in terms of metabolic response.

An essential aspect is that the measurements are performed over about 1 min time window. In many cases, it can therefore be argued that the biochemical machinery of the organism will not substantially change on such a short time scale, especially due to the transient nature of the bolus injection. This does obviously not mean that the organism is not affected by the injection, and the supraphysiological amount of injected molecules will have to be cleared off from the blood pool. It can however be 
reasonably assumed that the one-minute window represents a snapshot of the situation prior to injection.

The usual question asked by biologists and biochemists interested in this technology is the following: can we possibly reduce the doses to match physiological concentration? The short answer is yes, at least for a certain number of substrates and types of measurement. It is fundamental to understand that the substrate can usually be observed at physiological concentration, but the resulting metabolites are often in too low concentration to be detected. The conversion rates will indeed depend on the uptake and metabolic fluxes of a particular organ or cells, in short on the concentration of transporters and enzymes and their affinity to the substrate and intermediate metabolites. Therefore, in specific cells or organs that take up large amounts of substrates, like the heart or the liver, it is possible to measure metabolism with low injection doses.

The associated question is therefore to determine what substrates will provide enough signal to perform metabolic measurements at physiological levels. This will obviously depend on the future technological improvements in terms of polarization and detection sensitivity. In the author's opinion, it will eventually be possible to perform in vivo preclinical hyperpolarized MR experiments at physiological level with at least a few substrates such as lactate, glucose, and possibly pyruvate. In order to reach the necessary sensitivity for such experiments, the three key points (a-c) presented in the previous sections (a-c) will have to be optimized. Hyperpolarized MR with substrates such as ${ }^{13} \mathrm{C}$-glucose could then possibly become a widely available modality. There is little doubt that this technology would be highly appealing to most researchers 
working with animal models on the understanding of diseases and the development of treatments.

\section{Conclusion}

Although hyperpolarized MR via dissolution DNP is based on well-known principles, the technology is still new and only a relatively small number of laboratories can perform in vivo hyperpolarized MR experiments in a routine manner. This is because it not only requires skilled and trained researchers for optimal data acquisition, the MR sequences not being always widely available or easily implementable on all platforms, but also researchers dedicated to hardware optimization. The latter includes in particular the implementation of sensitive rf coils, the commercially available equipment being currently limited. The prerequisite for a wider dissemination of this technology for preclinical applications is the implementation of a highly reproducible and overall simplified methodology to allow standardized experiments by a large number of MR researchers and technicians. This translates into the need for a higher level of automation and some simplifications. The development of the HyperSense by Oxford Instruments was a first step towards the dissemination of the hyperpolarized MR method for preclinical applications since the automated dissolution process alleviate the need for an in-house expert in cryogenics. The author's research group developed, at the Ecole Polytechnique Fédérale de Lausanne (EPFL), a way to automate sample transfer and injection, allowing to do more with less initial polarization. Using this method, it was demonstrated that many in vivo preclinical studies can be performed with no more than $10 \%{ }^{13} \mathrm{C}$ polarization with a large variety of compounds. It would however be desirable to further increase the liquid-state 
polarization up to at least $50 \%$ at the time of injection in order to eventually perform high-resolution imaging.

In conclusion, because of the high potential of the technology and its promises for clinical applications demonstrated by the recent in-human trial [73], it is expected that new dedicated dissolution DNP setups for preclinical applications will be developed in the near future. It is the author's opinion that if the novel technologies are simpler and more reliable, hyperpolarized MR by dissolution DNP could become a standard and powerful tool for preclinical studies in the field of oncology, cardiology, hepatology, neuroscience, and many others.

\section{ACKNOWLEDGMENTS}

The author declares no competing financial interests. The author was supported by the Swiss National Science Foundation (grant PP00P2_ 157547). 


\section{REFERENCES}

[1] M.S. Albert, G.D. Cates, B. Driehuys, W. Happer, B. Saam, C.S. Springer, A. Wishnia, Biological Magnetic-Resonance-Imaging Using Laser Polarized Xe-129, Nature 370 (1994) 199-201.

[2] J.H. Ardenkjaer-Larsen, B. Fridlund, A. Gram, G. Hansson, L. Hansson, M.H. Lerche, R. Servin, M. Thaning, K. Golman, Increase in signal-to-noise ratio of $>10,000$ times in liquid-state NMR, Proc. Natl. Acad. Sci. U.S.A. 100 (2003) 10158-10163.

[3] G. Frossati, Polarization of He-3, D-2 and (eventually) Xe-129 using low temperatures and high magnetic fields, J. Low Temp. Phys. 111 (1998) 521-532.

[4] C.R. Bowers, D.P. Weitekamp, Transformation of Symmetrization Order to Nuclear-Spin Magnetization by Chemical-Reaction and Nuclear-MagneticResonance, Phys. Rev. Lett. 57 (1986) 2645-2648.

[5] C.R. Bowers, D.P. Weitekamp, Para-Hydrogen and Synthesis Allow Dramatically Enhanced Nuclear Alignment, J. Am. Chem. Soc. 109 (1987) 55415542.

[6] K. Golman, O. Axelsson, H. Johannesson, S. Mansson, C. Olofsson, J.S. Petersson, Parahydrogen-induced polarization in imaging: Subsecond C-13 angiography, Magn. Reson. Med. 46 (2001) 1-5.

[7] S. Gloggler, J. Colell, S. Appelt, Para-hydrogen perspectives in hyperpolarized NMR, J. Magn. Reson. 235 (2013) 130-142.

[8] S. Hu, P.E. Larson, M. Vancriekinge, A.M. Leach, I. Park, C. Leon, J. Zhou, P.J. Shin, G. Reed, P. Keselman, C. von Morze, H. Yoshihara, R.A. Bok, S.J. Nelson, J. Kurhanewicz, D.B. Vigneron, Rapid sequential injections of hyperpolarized [1-(13)C]pyruvate in vivo using a sub-kelvin, multi-sample DNP polarizer, Magn. Reson. Imaging 31 (2013) 490-496.

[9] H.A.I. Yoshihara, E. Can, M. Karlsson, M.H. Lerche, J. Schwitter, A. Comment, High-field dissolution dynamic nuclear polarization of [1-13C]pyruvic acid, submitted.

[10] L. Lumata, S.J. Ratnakar, A. Jindal, M. Merritt, A. Comment, C. Malloy, A.D. Sherry, Z. Kovacs, BDPA: An Efficient Polarizing Agent for Fast Dissolution Dynamic Nuclear Polarization NMR Spectroscopy, Chemistry 17 (2011) 10825-10827.

[11] J.A. Bastiaansen, T. Cheng, H. Lei, R. Gruetter, A. Comment, Direct noninvasive estimation of myocardial tricarboxylic acid cycle flux in vivo using hyperpolarized C magnetic resonance, J. Mol. Cell. Cardiol. 87 (2015) 129-137.

[12] J.M. Bastiaansen, H.I. Yoshihara, Y. Takado, R. Gruetter, A. Comment, Hyperpolarized 13C lactate as a substrate for in vivo metabolic studies in skeletal muscle, Metabolomics 10 (2014) 986-994.

[13] M. Mishkovsky, A. Comment, R. Gruetter, In vivo detection of brain Krebs cycle intermediate by hyperpolarized magnetic resonance, J. Cereb. Blood Flow Metab. 32 (2012) 2108-2113.

[14] A. Capozzi, J.-N. Hyacinthe, T. Cheng, T.R. Eichhorn, G. Boero, C. Roussel, J.J. van der Klink, A. Comment, Photoinduced Nonpersistent Radicals as Polarizing Agents for X-Nuclei Dissolution Dynamic Nuclear Polarization, J. Phys. Chem. C 119 (2015) 22632-22639.

[15] T.R. Eichhorn, Y. Takado, N. Salameh, A. Capozzi, T. Cheng, J.N. Hyacinthe, M. Mishkovsky, C. Roussel, A. Comment, Hyperpolarization without 
persistent radicals for in vivo real-time metabolic imaging, Proc. Natl. Acad. Sci. U.S.A. 110 (2013) 18064-18069.

[16] S. Jannin, A. Comment, F. Kurdzesau, J.A. Konter, P. Hautle, B. van den Brandt, J.J. van der Klink, A $140 \mathrm{GHz}$ prepolarizer for dissolution dynamic nuclear polarization, J. Chem. Phys. 128 (2008) 241102.

[17] H. Johanneson, S. Macholl, J.H. Ardenkjaer-Larsen, Dynamic Nuclear Polarization of [1-(13)C]pyruvic acid at 4.6 tesla, J. Magn. Reson. 197 (2009) 167-175.

[18] T. Cheng, A. Capozzi, Y. Takado, R. Balzan, A. Comment, Over 35\% liquid-state 13C polarization obtained via dissolution dynamic nuclear polarization at $7 \mathrm{~T}$ and $1 \mathrm{~K}$ using ubiquitous nitroxyl radicals, Phys. Chem. Chem. Phys. 15 (2013) 20819-20822.

[19] A. Comment, B. van den Brandt, K. Uffmann, F. Kurdzesau, S. Jannin, J.A. Konter, P. Hautle, W.T. Wenckebach, R. Gruetter, J.J. van der Klink, Design and performance of a DNP prepolarizer coupled to a rodent MRI scanner, Concepts Magn. Reson. 31B (2007) 255-269.

[20] K.M. Brindle, Imaging Metabolism with Hyperpolarized C-13-Labeled Cell Substrates, J. Am Chem. Soc. 137 (2015) 6418-6427.

[21] A. Comment, M.E. Merritt, Hyperpolarized Magnetic Resonance as a Sensitive Detector of Metabolic Function, Biochemistry 53 (2014) 7333-7357.

[22] L. Salamanca-Cardona, K. Keshari, 13 C-labeled biochemical probes for the study of cancer metabolism with dynamic nuclear polarization-enhanced magnetic resonance imaging, Cancer \& Metabolism 3 (2015) 9.

[23] H.M. Zhang, The Potential of Hyperpolarized C-13 MRI in Assessing Signaling Pathways in Cancer, Acad. Radiol. 21 (2014) 215-222.

[24] T.B. Rodrigues, E.M. Serrao, B.W.C. Kennedy, D.-E. Hu, M.I. Kettunen, K.M. Brindle, Magnetic resonance imaging of tumor glycolysis using hyperpolarized 13C-labeled glucose, Nat. Med. 20 (2014) 93-97.

[25] S. Meier, P.R. Jensen, J.O. Duus, Real-time detection of central carbon metabolism in living Escherichia coli and its response to perturbations, FEBS Lett. 585 (2011) 3133-3138.

[26] S. Meier, M. Karlsson, P.R. Jensen, M.H. Lerche, J.O. Duus, Metabolic pathway visualization in living yeast by DNP-NMR, Mol. Biosyst. 7 (2011) 28342836.

[27] C.E. Christensen, M. Karlsson, J.R. Winther, P.R. Jensen, M.H. Lerche, Non-invasive in-cell determination of free cytosolic [NAD+]/[NADH] ratios using hyperpolarized glucose show large variations in metabolic phenotypes, J. Biol. Chem. 289 (2014) 2344-2352.

[28] T. Harris, H. Degani, L. Frydman, Hyperpolarized C-13 NMR studies of glucose metabolism in living breast cancer cell cultures, NMR Biomed. 26 (2013) 1831-1843.

[29] J. Kurhanewicz, D.B. Vigneron, K. Brindle, E.Y. Chekmenev, A. Comment, C.H. Cunningham, R.J. DeBerardinis, G.G. Green, M.O. Leach, S.S. Rajan, R.R. Rizi, B.D. Ross, W.S. Warren, C.R. Malloy, Analysis of Cancer Metabolism by Imaging Hyperpolarized Nuclei: Prospects for Translation to Clinical Research, Neoplasia 13 (2011) 81-97.

[30] M.G. Vander Heiden, L.C. Cantley, C.B. Thompson, Understanding the Warburg Effect: The Metabolic Requirements of Cell Proliferation, Science 324 (2009) 1029-1033. 
[31] O. Warburg, K. Posener, E. Negelein, On the metabolism of carcinoma cells., Biochem. Z. 152 (1924) 309-344.

[32] F.A. Gallagher, S.E. Bohndiek, M.I. Kettunen, D.Y. Lewis, D. Soloviev, K.M. Brindle, Hyperpolarized C-13 MRI and PET: In Vivo Tumor Biochemistry, J. Nucl. Med. 52 (2011) 1333-1336.

[33] H. Gutte, A.E. Hansen, S.T. Henriksen, H.H. Johannesen, J. ArdenkjaerLarsen, A. Vignaud, A.E. Hansen, B. Børresen, T.L. Klausen, A.-M.N. Wittekind, N. Gillings, A.T. Kristensen, A. Clemmensen, L. Højgaard, A. Kjær, Simultaneous hyperpolarized (13)C-pyruvate MRI and (18)F-FDG-PET in cancer (hyperPET): feasibility of a new imaging concept using a clinical PET/MRI scanner, Am. J. Nucl. Med. Mol. Imaging, 5 (2015) 38-45.

[34] M.I. Menzel, E.V. Farrell, M.A. Janich, O. Khegai, F. Wiesinger, S. Nekolla, A.M. Otto, A. Haase, R.F. Schulte, M. Schwaiger, Multimodal Assessment of In Vivo Metabolism with Hyperpolarized [1-C-13]MR Spectroscopy and F-18-FDG PET Imaging in Hepatocellular Carcinoma Tumor-Bearing Rats, J. Nucl. Med. 54 (2013) 1113-1119.

[35] R.A. Cairns, I.S. Harris, T.W. Mak, Regulation of cancer cell metabolism, Nat. Rev. Cancer 11 (2011) 85-95.

[36] K.X. Moreno, S. Satapati, R.J. DeBerardinis, S.C. Burgess, C.R. Malloy, M.E. Merritt, Real-time Detection of Hepatic Gluconeogenic and Glycogenolytic States Using Hyperpolarized [2-C-13]Dihydroxyacetone, J. Biol. Chem. 289 (2014) 35859-35867.

[37] R.B. van Heeswijk, K. Uffmann, A. Comment, F. Kurdzesau, C. Perazzolo, C. Cudalbu, S. Jannin, J.A. Konter, P. Hautle, B. van den Brandt, G. Navon, J.J. van der Klink, R. Gruetter, Hyperpolarized Lithium-6 as a Sensor of Nanomolar Contrast Agents, Magn. Reson. Med. 61 (2009) 1489-1493.

[38] K. Golman, J.H. Ardenkjaer-Larsen, J.S. Petersson, S. Mansson, I. Leunbach, Molecular imaging with endogenous substances, Proc. Natl. Acad. Sci. U.S.A. 100 (2003) 10435-10439.

[39] K. Golman, J.H. Ardenkjaer-Larsen, J. Svensson, O. Axelsson, G. Hansson, L. Hansson, H. Johannesson, I. Leunbach, S. Mansson, J.S. Petersson, G. Pettersson, R. Servin, L.G. Wistrand, C-13-angiography, Acad. Radiol. 9 (2002) S507-S510.

[40] A.K. Grant, E. Vinogradov, X.E. Wang, R.E. Lenkinski, D.C. Alsop, Perfusion Imaging with a Freely Diffusible Hyperpolarized Contrast Agent, Magn. Reson. Med. 66 (2011) 746-755.

[41] G.D. Reed, C. von Morze, R. Bok, B.L. Koelsch, M. Van Criekinge, K.J. Smith, H. Shang, P.E.Z. Larson, J. Kurhanewicz, D.B. Vigneron, High Resolution C-13 MRI With Hyperpolarized Urea: In Vivo T-2 Mapping and N15 Labeling Effects, IEEE T. Med. Imaging, 33 (2014) 362-371.

[42] A. Comment, S. Jannin, J.N. Hyacinthe, P. Mieville, R. Sarkar, P. Ahuja, P.R. Vasos, X. Montet, F. Lazeyras, J.P. Vallee, P. Hautle, J.A. Konter, B. van den Brandt, J.P. Ansermet, R. Gruetter, G. Bodenhausen, Hyperpolarizing Gases via Dynamic Nuclear Polarization and Sublimation, Phys. Rev. Lett. 105 (2010).

[43] B. Driehuys, H.E. Möller, Z.I. Cleveland, J. Pollaro, L.W. Hedlund, Pulmonary Perfusion and Xenon Gas Exchange in Rats: MR Imaging with Intravenous Injection of Hyperpolarized 129Xe, Radiology 252 (2009) 386-393.

[44] J.H. Ardenkjaer-Larsen, I. Laursen, I. Leunbach, G. Ehnholm, L.G. Wistrand, J.S. Petersson, K. Golman, EPR and DNP properties of certain novel 
single electron contrast agents intended for oximetric imaging, J. Magn. Reson. 133 (1998) 1-12.

[45] M. Batel, M. Krajewski, K. Weiss, O. With, A. Dapp, A. Hunkeler, M. Gimersky, K.P. Pruessmann, P. Boesiger, B.H. Meier, S. Kozerke, M. Ernst, A multi-sample 94 GHz dissolution dynamic-nuclear-polarization system, J. Magn. Reson. 214 (2012) 166-174.

[46] F. Kurdzesau, B. van den Brandt, A. Comment, P. Hautle, S. Jannin, J.J. van der Klink, J.A. Konter, Dynamic nuclear polarization of small labelled molecules in frozen water-alcohol solutions, J. Phys. D: Appl. Phys. 41 (2008).

[47] A. Bornet, R. Melzi, A.J.P. Linde, P. Hautle, B. van den Brandt, S. Jannin, G. Bodenhausen, Boosting Dissolution Dynamic Nuclear Polarization by Cross Polarization, J. Phys. Chem. Lett. 4 (2013) 111-114.

[48] J.H. Ardenkjaer-Larsen, A.M. Leach, N. Clarke, J. Urbahn, D. Anderson, T.W. Skloss, Dynamic Nuclear Polarization Polarizer for Sterile Use Intent, NMR Biomed. 24 (2011) 927-932.

[49] T. Cheng, M. Mishkovsky, J.A.M. Bastiaansen, O. Ouari, P. Hautle, P. Tordo, B. van den Brandt, A. Comment, Automated transfer and injection of hyperpolarized molecules with polarization measurement prior to in vivo NMR, NMR Biomed. 26 (2013) 1582-1588.

[50] H. Yoshihara, J.A.M. Bastiaansen, M. Karlsson, M.H. Lerche, A. Comment, J. Schwitter, Hyperpolarized [1-13C]octanoate: A Probe of Myocardial 及Oxidation, in: Proc. Intl. Soc. Mag. Reson. Med. 23, 91 (2015, Toronto, ON, Canada), Toronto, ON, Canada, 2015.

[51] M. Mishkovsky, Brian Anderson, A. Capozzi, R. Gruetter, Z. Kovacs, A. Comment, Probing cerebral glucose metabolism in vivo in real time, submitted.

[52] P. Mieville, P. Ahuja, R. Sarkar, S. Jannin, P.R. Vasos, S. Gerber-Lemaire, M. Mishkovsky, A. Comment, R. Gruetter, O. Ouari, P. Tordo, G. Bodenhausen, Scavenging Free Radicals To Preserve Enhancement and Extend Relaxation Times in NMR using Dynamic Nuclear Polarization, Angew. Chem. Int. Ed. 49 (2010) 6182-6185.

[53] T. Cheng, M. Mishkovsky, M. Junk, D. Hinderberger, K. Münnemann, A. Comment, Producing radical-free hyperpolarized solutions for in vivo magnetic resonance, Proc. Intl. Soc. Mag. Reson. Med. 20, 1664 (2012, Melbourne, Australia), 20 (2012).

[54] D. Gajan, A. Bornet, B. Vuichoud, J. Milani, R. Melzi, H.A. van Kalkeren, L. Veyre, C. Thieuleux, M.P. Conley, W.R. Gruning, M. Schwarzwalder, A. Lesage, C. Coperet, G. Bodenhausen, L. Emsley, S. Jannin, Hybrid polarizing solids for pure hyperpolarized liquids through dissolution dynamic nuclear polarization, Proc. Natl. Acad. Sci. U.S.A. 111 (2014) 14693-14697.

[55] J.A.M. Bastiaansen, T. Cheng, M. Mishkovsky, J.M.N. Duarte, A. Comment, R. Gruetter, In vivo enzymatic activity of acetylCoA synthetase in skeletal muscle revealed by $\mathrm{C}-13$ turnover from hyperpolarized [1-C-13]acetate to [1-C-13]acetylcarnitine, Biochim. Biophys. Acta Gen. Subj. 1830 (2013) 41714178.

[56] D.K. Hill, M.R. Orton, E. Mariotti, J.K.R. Boult, R. Panek, M. Jafar, H.G. Parkes, Y. Jamin, M.F. Miniotis, N.M.S. Al-Saffar, M. Beloueche-Babari, S.P. Robinson, M.O. Leach, Y.-L. Chung, T.R. Eykyn, Model Free Approach to Kinetic Analysis of Real-Time Hyperpolarized ${ }^{13} \mathrm{C}$ Magnetic Resonance Spectroscopy Data, PLOS ONE 8 (2013) e71996. 
[57] Y. Xing, G.D. Reed, J.M. Pauly, A.B. Kerr, P.E.Z. Larson, Optimal variable flip angle schemes for dynamic acquisition of exchanging hyperpolarized substrates, J. Magn. Reson. 234 (2013) 75-81.

[58] S. Hu, M. Lustig, A. Balakrishnan, P.E.Z. Larson, R. Bok, J. Kurhanewicz, S.J. Nelson, A. Goga, J.M. Pauly, D.B. Vigneron, 3D Compressed Sensing for Highly Accelerated Hyperpolarized C-13 MRSI With In Vivo Applications to Transgenic Mouse Models of Cancer, Magn. Reson. Med. 63 (2010) 312-321.

[59] A.P. Chen, C.H. Cunningham, Single voxel localization for dynamic hyperpolarized (13)C MR spectroscopy, J. Magn. Reson. 258 (2015) 81-85.

[60] M. Marjanska, I. Iltis, A.A. Shestov, D.K. Deelchand, C. Nelson, K. Ugurbil, P.G. Henry, In vivo $\mathrm{C}-13$ spectroscopy in the rat brain using hyperpolarized [1C-13]pyruvate and [2-C-13]pyruvate, J. Magn. Reson. 206 (2010) 210-218.

[61] M. Mishkovsky, T. Cheng, A. Comment, R. Gruetter, Localized in vivo hyperpolarization transfer sequences, Magn. Reson. Med. 68 (2012) 349-352.

[62] P.R. Jensen, T. Peitersen, M. Karlsson, R. In 't Zandt, A. Gisselsson, G. Hansson, S. Meier, M.H. Lerche, Tissue-specific short chain fatty acid metabolism and slow metabolic recovery after ischemia from hyperpolarized NMR in vivo, J. Biol. Chem. 284 (2009) 36077-36082.

[63] M.A. Schroeder, H.J. Atherton, M.S. Dodd, K. Clarke, G.K. Radda, D.J. Tyler, Real Time, in vivo Observation of Oxidative Carbohydrate Metabolism Reveals the Key Regulatory Role of Acetyl-Carnitine as a Substrate Buffer in the Heart, Circulation 120 (2009) S855-S856.

[64] C.H. Cunningham, A.P. Chen, M.J. Albers, J. Kurhanewicz, R.E. Hurd, Y.F. Yen, J.M. Pauly, S.J. Nelson, D.B. Vigneron, Double spin-echo sequence for rapid spectroscopic imaging of hyperpolarized C-13, J. Magn. Reson. 187 (2007) 357-362.

[65] P.E.Z. Larson, A.B. Kerr, A.P. Chen, M.S. Lustig, M.L. Zierhut, S. Hu, C.H. Cunningham, J.M. Pauly, J. Kurhanewicz, D.B. Vigneron, Multiband excitation pulses for hyperpolarized C-13 dynamic chemical-shift imaging, J. Magn. Reson. 194 (2008) 121-127.

[66] S. Mansson, J.S. Petersson, K. Scheffler, Fast metabolite mapping in the pig heart after injection of hyperpolarized 13C-pyruvate with low-flip angle balanced steady-state free precession imaging, Magn. Reson. Med. 68 (2012) 1894-1899.

[67] C. von Morze, G. Reed, P. Shin, P.E.Z. Larson, S. Hu, R. Bok, D.B. Vigneron, Multi-band frequency encoding method for metabolic imaging with hyperpolarized [1-C-13]pyruvate, J. Magn. Reson. 211 (2011) 109-113.

[68] R. Schmidt, C. Laustsen, J.N. Dumez, M.I. Kettunen, E.M. Serrao, I. MarcoRius, K.M. Brindle, J.H. Ardenkjaer-Larsen, L. Frydman, In vivo single-shot C13 spectroscopic imaging of hyperpolarized metabolites by spatiotemporal encoding, J. Magn. Reson. 240 (2014) 8-15.

[69] P. Cao, X. Zhang, I. Park, C. Najac, S.J. Nelson, S. Ronen, P.E. Larson, H$C$ independently tuned radiofrequency surface coil applied for in vivo hyperpolarized MRI, Magn. Reson. Med. (2015).

[70] H. Lim, K. Thind, F.M. Martinez-Santiesteban, T.J. Scholl, Construction and Evaluation of a Switch-Tuned C-13 - H-1 Birdcage Radiofrequency Coil for Imaging the Metabolism of Hyperpolarized C-13-Enriched Compounds, J. Magn. Reson. Imaging 40 (2014) 1082-1090.

[71] J. Wosik, K. Nesteruk, I.-C. Tan, K. Qin, J.A. Bankson, Cryogenic Receiveonly 7 Tesla Coil for MRI of Hyperpolarized 13C in: Proc. Intl. Soc. Mag. 
Reson. Med. 23, 1791 (2015, Toronto, Ontario, Canada), Toronto, Ontario, Canada, 2015.

[72] M.M. Chaumeil, P.E.Z. Larson, H.A.I. Yoshihara, O.M. Danforth, D.B. Vigneron, S.J. Nelson, R.O. Pieper, J.J. Phillips, S.M. Ronen, Non-invasive in vivo assessment of IDH1 mutational status in glioma, Nat. Commun. 4 (2013).

[73] S.J. Nelson, J. Kurhanewicz, D.B. Vigneron, P.E. Larson, A.L. Harzstark, M. Ferrone, M. van Criekinge, J.W. Chang, R. Bok, I. Park, G. Reed, L. Carvajal, E.J. Small, P. Munster, V.K. Weinberg, J.H. Ardenkjaer-Larsen, A.P. Chen, R.E. Hurd, L.I. Odegardstuen, F.J. Robb, J. Tropp, J.A. Murray, Metabolic Imaging of Patients with Prostate Cancer Using Hyperpolarized [113C]Pyruvate, Sci. Transl. Med. 5 (2013) 198 ra108.

[74] M. Kveder, D. Merunka, M. Jokic, J. Makarevic, B. Rakvin, Electron spinlattice relaxation in solid ethanol: Effect of nitroxyl radical hydrogen bonding and matrix disorder, Phys. Rev. B 80 (2009).

[75] M. Filibian, S.C. Serra, M. Moscardini, A. Rosso, F. Tedoldi, P. Carretta, The role of the glassy dynamics and thermal mixing in the dynamic nuclear polarization and relaxation mechanisms of pyruvic acid, Phys. Chem. Chem. Phys. 16 (2014) 27025-27036.

[76] L. Lumata, Z. Kovacs, A.D. Sherry, C. Malloy, S. Hill, J. van Tol, L. Yu, L.K. Song, M.E. Merritt, Electron spin resonance studies of trityl OX063 at a concentration optimal for DNP, Phys. Chem. Chem. Phys. 15 (2013) 9800-9807.

[77] J.A.M. Bastiaansen, M.E. Merritt, A. Comment, Measuring changes in substrate utilization in the myocardium in response to fasting using hyperpolarized [1-13C]butyrate and [1-13C]pyruvate, submitted. 


\section{FIGURE LEGENDS}

Figure 1. Theoretical ${ }^{13} \mathrm{C}$ (red curve) and electron (blue curve) spin polarization at 5 $\mathrm{T}$ as a function of temperature.

Figure 2. Electron spin longitudinal relaxation time $\left(\mathrm{T}_{1 \mathrm{e}}\right)$ for nitroxyl radicals at 0.35 $\mathrm{T}$ calculated as a function of temperature from the expression given in [74]. Note that $\mathrm{T}_{1 \mathrm{e}}$ is expected to have a similar temperature dependence at higher magnetic field and for other types of radicals $[75,76]$.

Figure 3. (a) Glycolytic metabolism in subcutaneously implanted xenografts of EL4 and LL2 cancer cell lines as detected with hyperpolarized $\left[\mathrm{U}_{-}^{2} \mathrm{H}, \mathrm{U}-{ }^{13} \mathrm{C}\right] \mathrm{glucose}$. Note the lack of lactate signal detected in normal tissues. (b) Images of glycolytic metabolism in the EL4 cancer model. Courtesy of Kevin M. Brindle.

Figure 4. Evolution of the signal intensity of the metabolites derived from [2${ }^{13} \mathrm{C}$ ]dihydroxyacetone measured in the perfused mouse liver in the glycogenolytic state. The metabolites are those associated with glycogenolysis. Pyr, pyruvate; PEP, phosphoenolpyruvate; $3 P G, 3$-phosphoglycerate; $G A 3 P$, glyceraldehyde 3-phosphate; Gly, glycerol; G3P, glycerol 3-phosphate; Lac, lactate; Ala, alanine. Courtesy of Matthew E. Merritt.

Figure 5. Projection imaging at $0.94 \mathrm{~mm}$ in-plane resolution. Top: localizer and $\left[{ }^{13} \mathrm{C},{ }^{15} \mathrm{~N}_{2}\right]$ urea images acquired with a $9 \mathrm{~cm}$ field of view (FOV). Bottom: localizer and $\left[{ }^{13} \mathrm{C},{ }^{15} \mathrm{~N}_{2}\right]$ urea images acquired over a 9 x $18 \mathrm{~cm}$ FOV. The kidneys were slightly anterior and posterior of the localizer slice. The $\left[{ }^{13} \mathrm{C},{ }^{15} \mathrm{~N}_{2}\right]$ urea image clearly depicts the aorta and its inferior branches. Virtually all $\left[{ }^{13} \mathrm{C},{ }^{15} \mathrm{~N}_{2}\right]$ urea images showed signal through the femoral arteries with some enhancement in the knee. Spinal vasculature is also depicted in the bottom $\left[{ }^{13} \mathrm{C},{ }^{15} \mathrm{~N}_{2}\right]$ urea image. Courtesy of Daniel B. Vigneron.

Figure 6. DNP polarizer for sterile use intent. Reproduced with permission from [48]. 
Figure 7. Sketch of the dissolution DNP setup installed at the Center for Biomedical Imaging (CIBM) of the Ecole Polytechnique Fédérale de Lausanne (EPFL). A 5 T, 1 $\mathrm{K}$ hyperpolarizer is located $\sim 4 \mathrm{~m}$ from a $9.4 \mathrm{~T}$ rodent MR scanner. A specific device minimizing the delay between the dissolution and the infusion of DNP-enhanced molecules has been implemented for in vivo applications.

Figure 8. Theoretical relative signal-to-noise ratio (SNR) corresponding to the sum of all hyperpolarized signals obtained after a series of single-pulse-acquire experiments separated by a given delay $\tau$ as a function of the pulse flip angle. The longitudinal relaxation time was set to $9 \mathrm{~s}$ to simulate the in vivo situation with hyperpolarized [U${ }^{2} \mathrm{H}, \mathrm{U}-{ }^{13} \mathrm{C}$ ]glucose [24].

Figure 9. Non-invasive in vivo assessment of IDH1 mutational status using hyperpolarized $\left[1-{ }^{13} \mathrm{C}\right] \alpha$-ketoglutarate in glioma (a) $\mathrm{T}_{2}$-weighted anatomical MR image of the head of a U87IDHmut (top) and a U87IDHwt (bottom) tumor-bearing animal overlaid with the grid used for $2 \mathrm{D}{ }^{13} \mathrm{C}$ CSI acquisition. The tumors appear as hyperintense regions. (b) Corresponding heatmap of hyperpolarized $\left[1-{ }^{13} \mathrm{C}\right] \alpha-\mathrm{KG}$ at $20 \mathrm{~s}$ post injection. (c) Corresponding heatmap of hyperpolarized $\left[1-{ }^{13} \mathrm{C}\right] 2-\mathrm{HG}$ at $20 \mathrm{~s}$ post injection, illustrating the presence of this metabolite in IDH1 mutant tumors only. Reprinted by permission from Macmillan Publishers Ltd: Nature Communications [72], copyright (2013).

Figure 10. In vivo ${ }^{13} \mathrm{C}$ spectrum (sum of 20 spectra) recorded in a rat heart following the co-injection and metabolism of hyperpolarized $\left[1-{ }^{13} \mathrm{C}\right]$ butyrate and $[1-$ ${ }^{13}$ C]pyruvate [77]. 


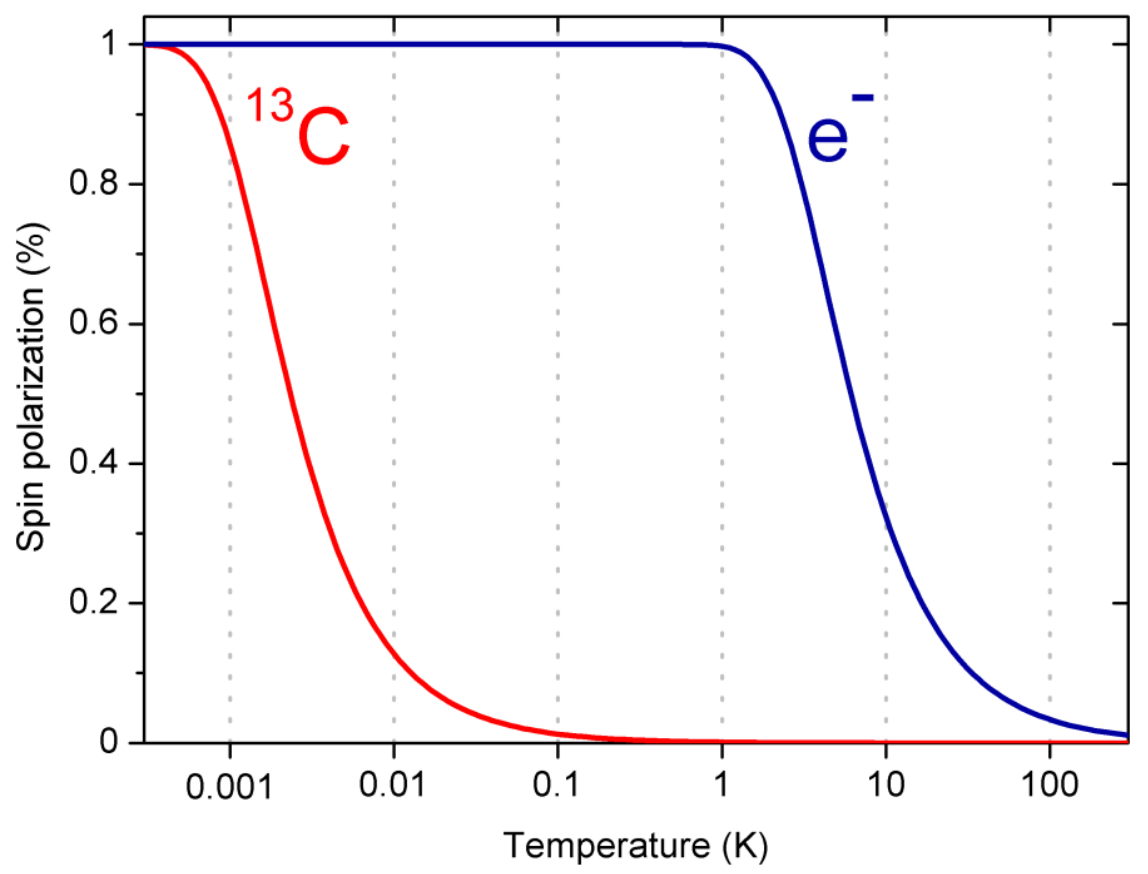




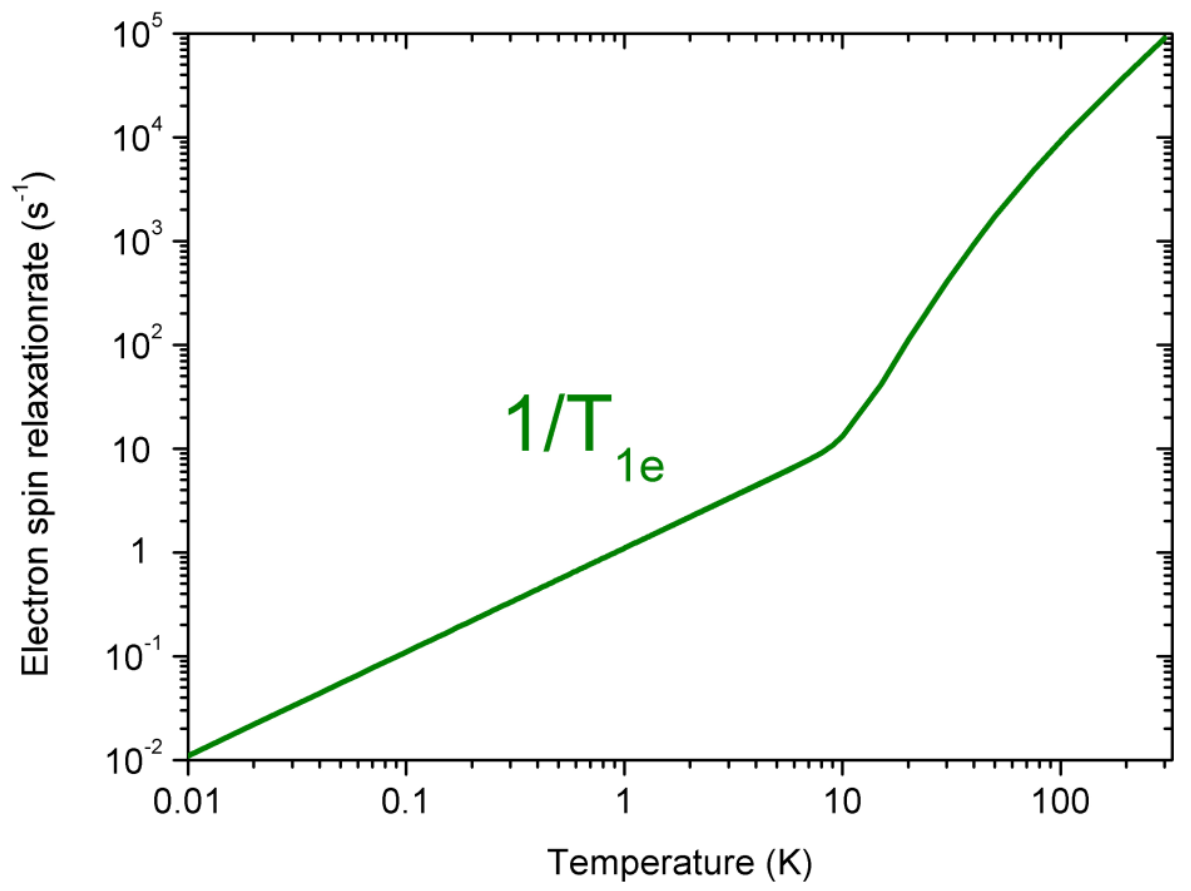


a

Glucose

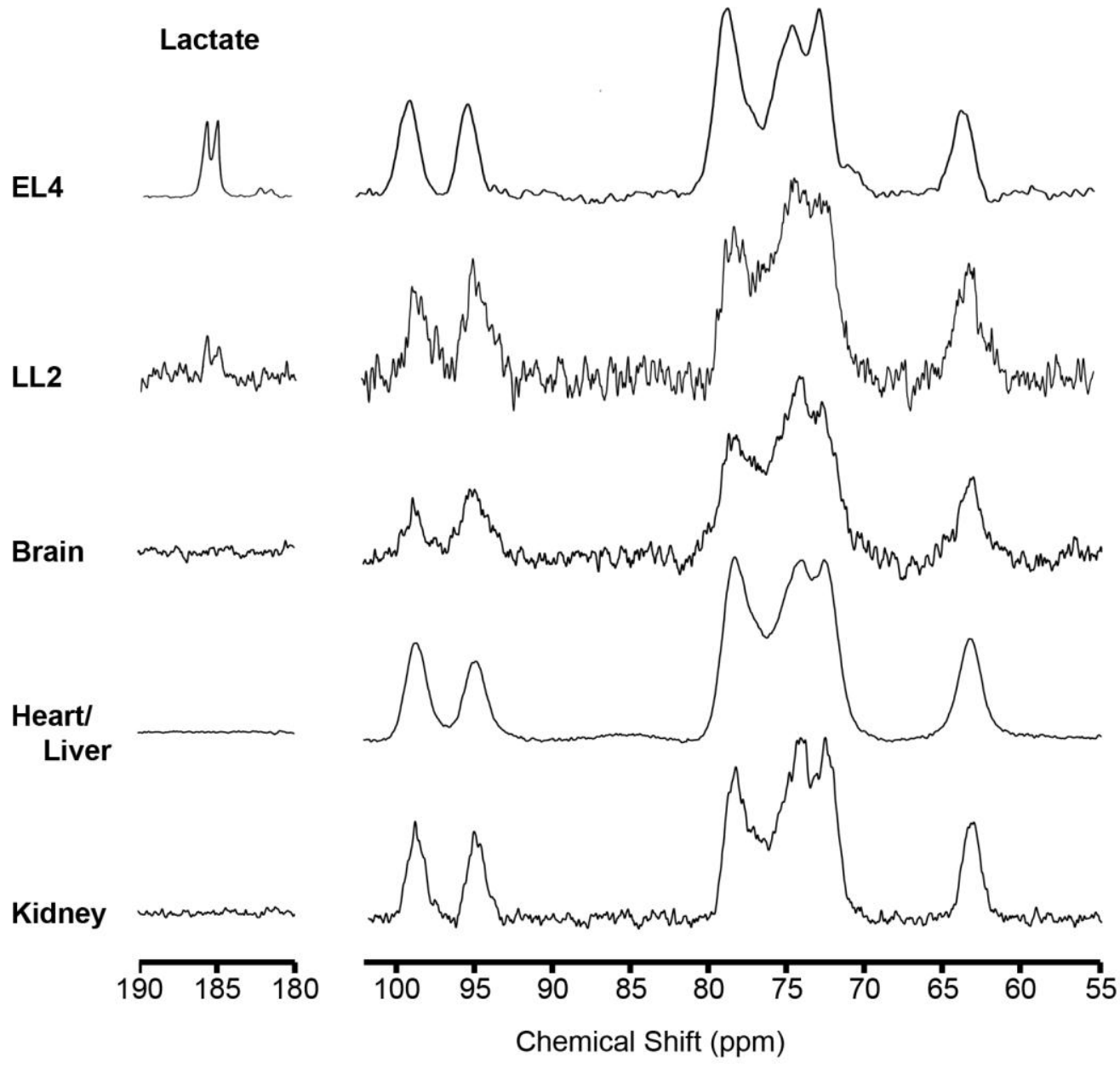

b

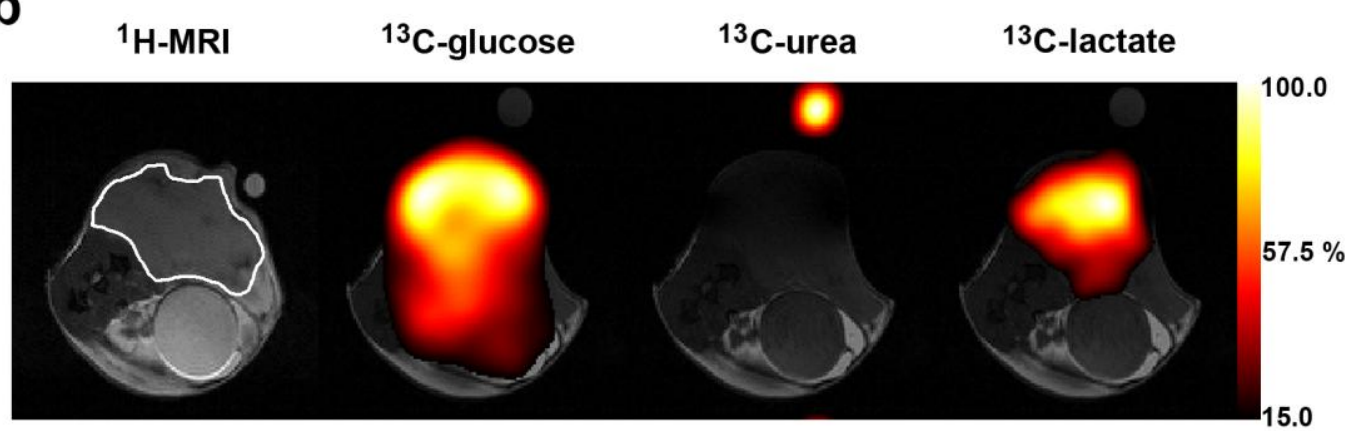




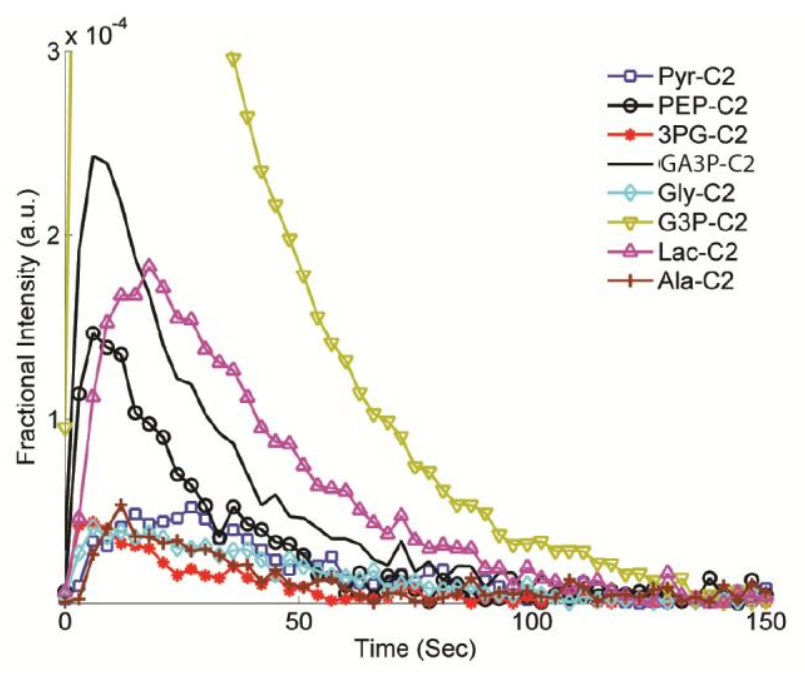




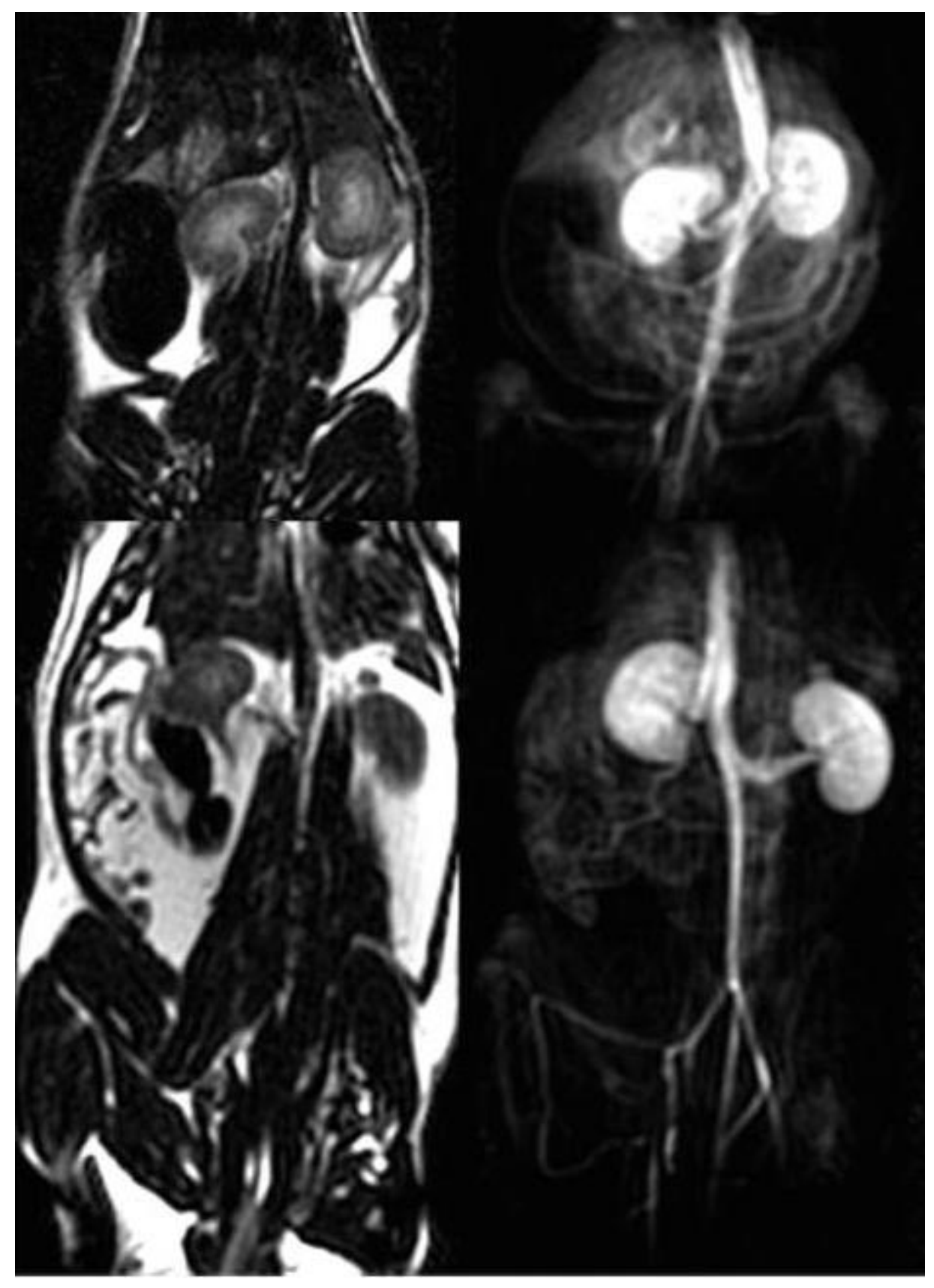




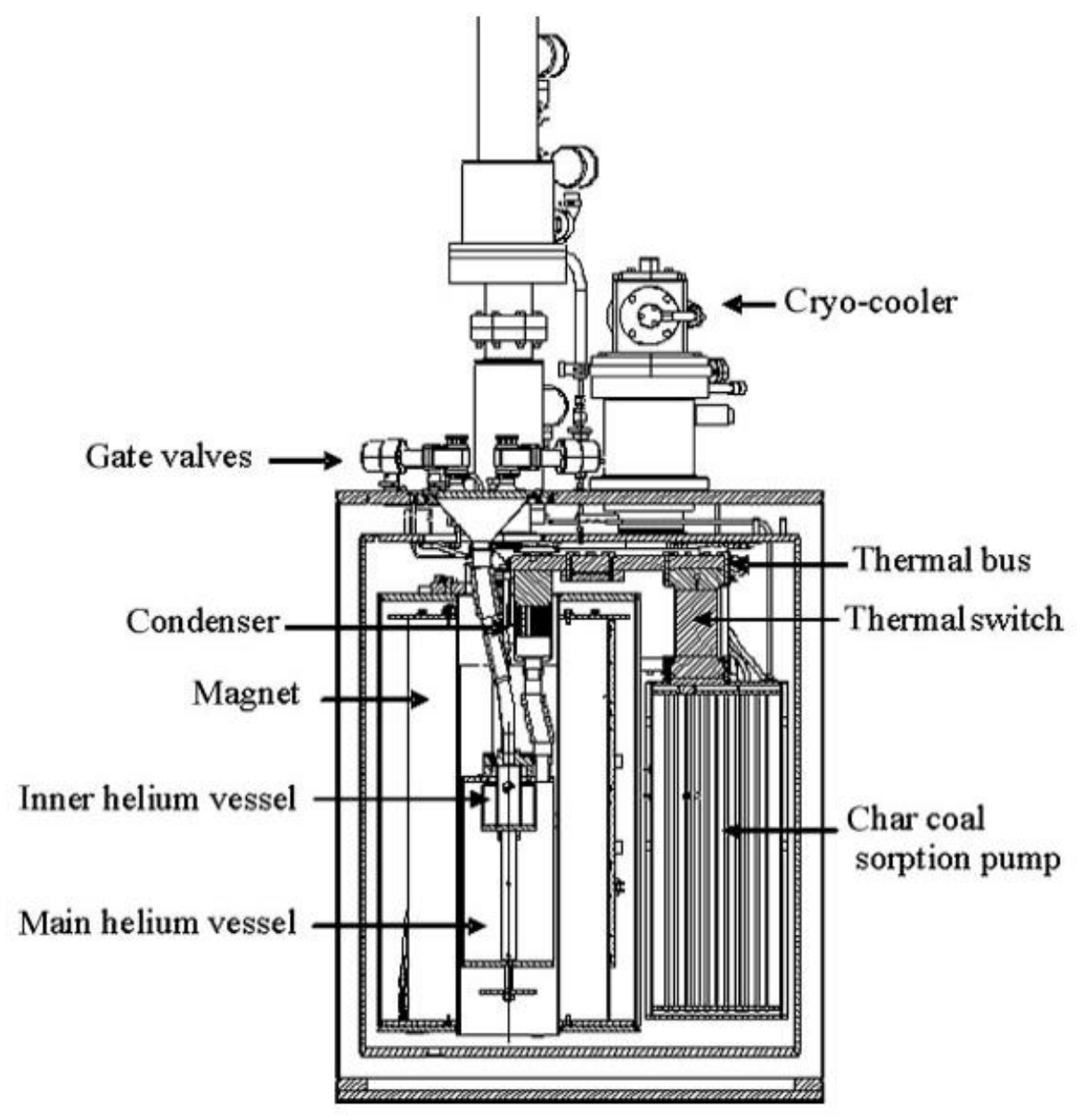




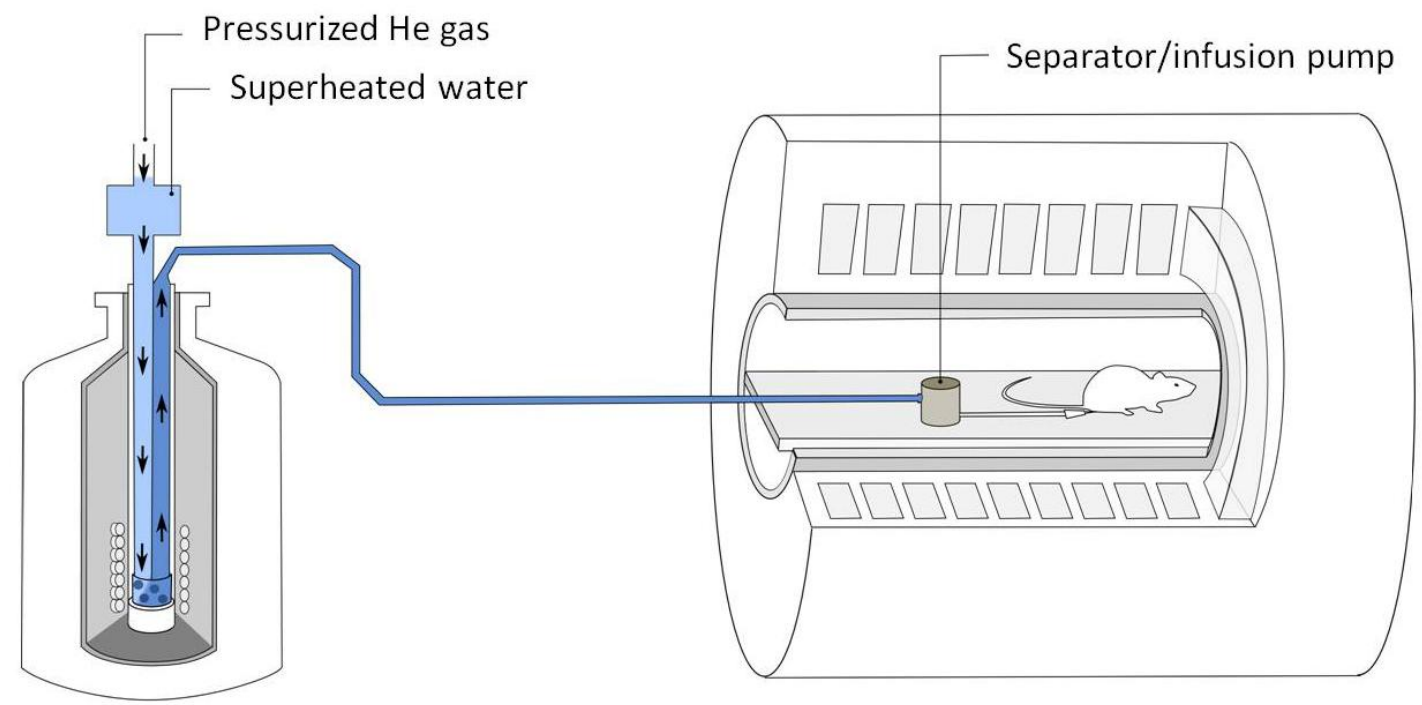




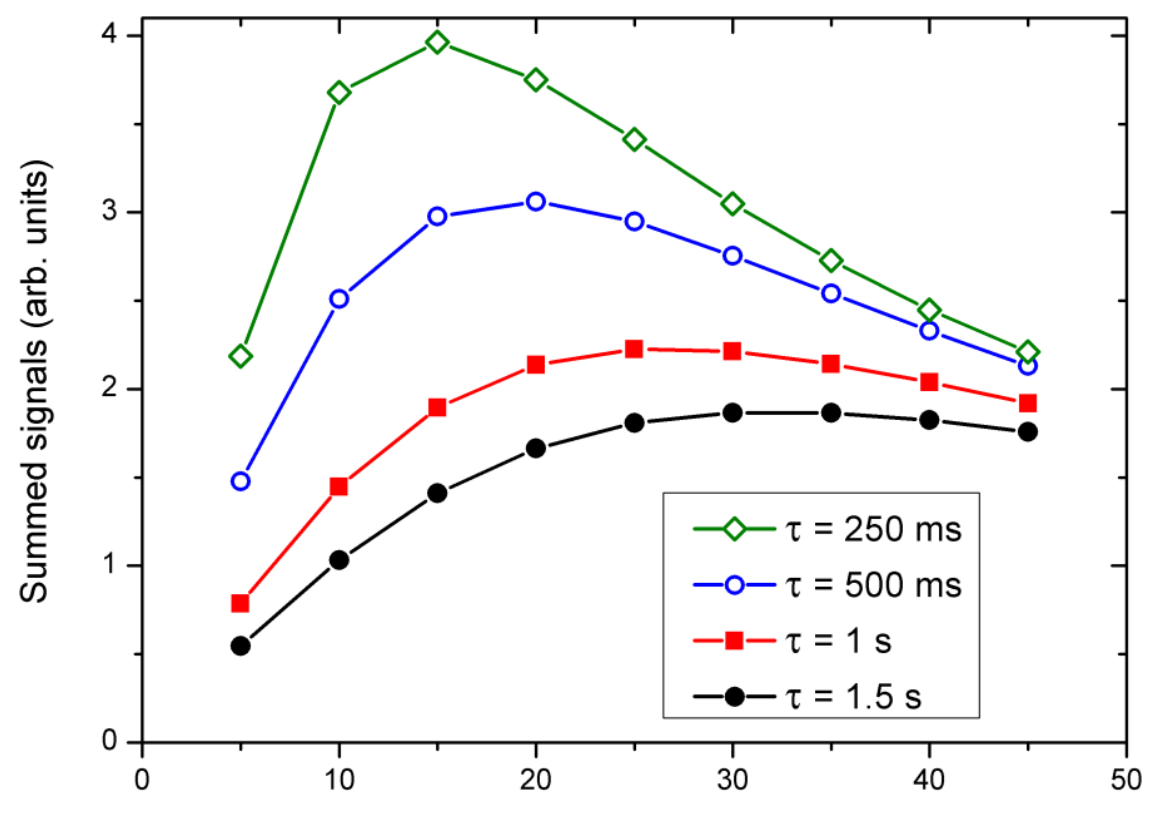

Pulse angle $\left({ }^{\circ}\right)$ 


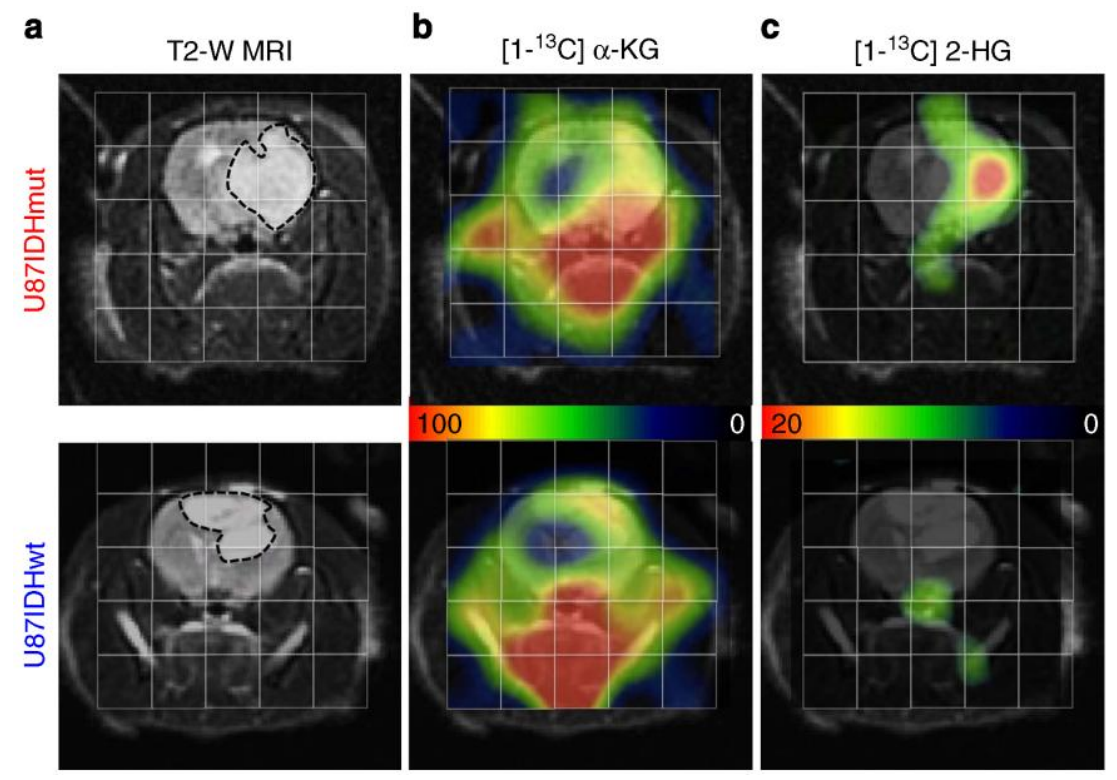




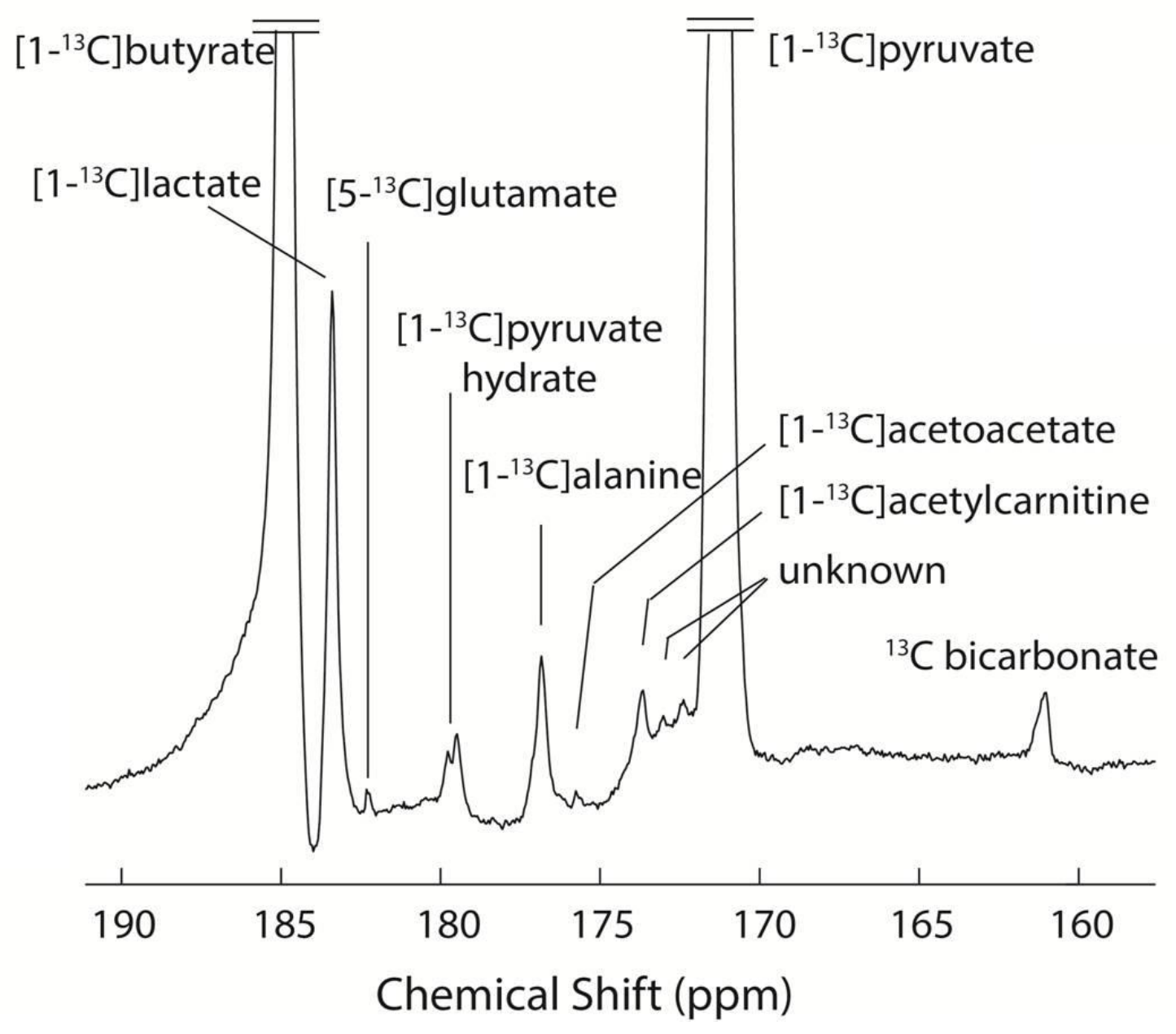




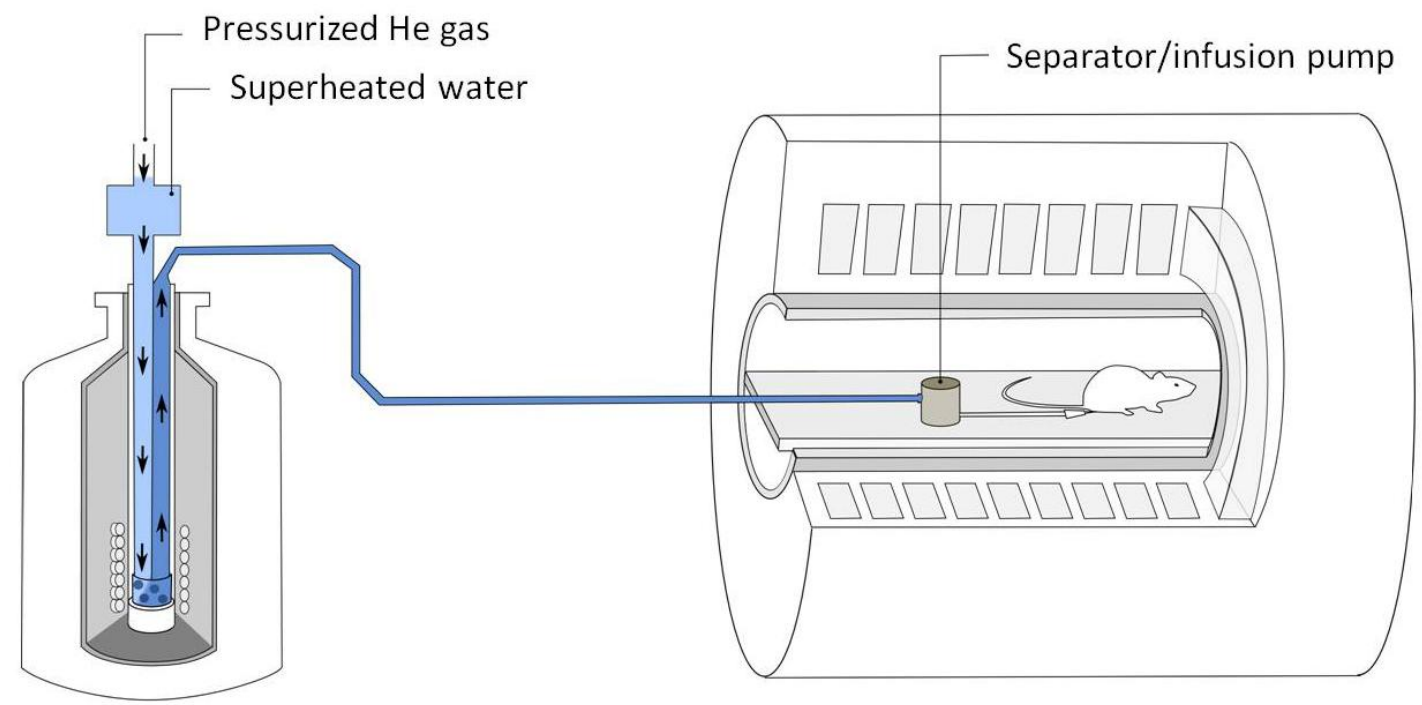

